\title{
Impact of Disability, Psychological Status, and Comorbidity on Health-Related Quality of Life Perceived by Subjects with Obesity
}

\author{
Lorenzo Maria Donini $^{a} \quad$ Aldo Rosano $^{b}$ Luca Di Lazzaro $^{a} \quad$ Carla Lubrano $^{a}$ \\ Mariagrazia Carbonellic Alessandro Pinto ${ }^{a}$ Anna Maria Giustia \\ Andrea Lenzi $^{\mathrm{a}}$ Mario Siervo $^{\mathrm{d}}$ \\ ${ }^{a}$ Medical Pathophysiology, Food Science and Endocrinology Section, Department of \\ Experimental Medicine, Sapienza University of Rome, Rome, Italy; ${ }^{b}$ Italian National Agency \\ for Regional Healthcare Services (AGENAS), Rome, Italy; ' San Camillo-Forlanini Hospital, \\ Rome, Italy; ${ }^{\mathrm{d}}$ Division of Physiology, Pharmacology and Neuroscience, School of Life \\ Sciences, University of Nottingham, Nottingham, UK
}

\section{Keywords}

Quality of life · Obesity · Disability

\begin{abstract}
Obesity is a rapidly growing public health problem affecting an increasing number of countries worldwide and creating substantial financial and health burdens. Obesity has a negative impact on health-related quality of life (HRQoL) with reference to physical and mental health status, social relationships, and economic factors. The aim of the present study is to investigate the association of number of comorbidities, psychological status, and disability level with HRQoL in a sample of subjects with obesity. Methods: A total of 273 subjects with obesity (199 women and 74 men) were recruited. Medical history and anthropometric measurements were carried out. The Italian version of the Laval questionnaire, the TSD-OC (SIO test for obesity-related disabilities), and SCL-90 (Symptom Checklist-90) tests were administered. The association between HRQoL (global and different domains scores of the Laval questionnaire dependent variable) and age, body mass index (BMI), comorbidity, TSD-OC, and SCL-90 was analysed using a stepwise linear regression model. Results: BMI, disability (TSD-OC global score), and psychological symptoms (SCL-90 global severity index) were found to be the main determinants of HRQoL. Single domains of HRQoL (symptoms, activity/mobility, personal hygiene/clothing, emotions, social interaction, and sexual life) showed different patterns of as-
\end{abstract}


Donini et al.: HRQoL Perceived by Subjects with Obesity

sociations with each domain of the Laval questionnaire. BMI, pain, and social life disruptions were found to be significantly associated with most of the HRQoL domains while age, comorbidities, psychological problems (depression, interpersonal symptoms, somatization), and disability were associated with only some domains of the Laval questionnaire. Education, psychological symptoms (obsessive-compulsive, anxiety, hostility, phobic anxiety, paranoid ideation), and disability domains (stiffness, housework, outdoor activities, occupational activities) were not associated with any of the dimensions of the Laval questionnaire. Discussion/Conclusion: The present study identified key determinants of QoL in subjects with obesity, which could help in refining the multidimensional diagnostic assessment of obesity as well as designing more effective interventions to improve HRQoL in these patients.

(C) 2020 The Author(s)

Published by S. Karger AG, Basel

\section{Introduction}

Obesity is a rapidly growing public health problem affecting an increasing number of countries worldwide and creating substantial financial and health burdens. According to the World Health Organization (WHO) obesity has nearly tripled since 1975; in 2016, more than 1.9 billion adults were overweight (of these over 650 million were obese) with prevalence estimates at $39 \%$ for overweight and $13 \%$ for obesity. It is also now established that most of the world's population live in countries where overweight and obesity kill more people than underweight [1].

A recent overview of 12 meta-analyses/reviews confirmed the significant and negative impact that obesity has on health-related quality of life (HRQoL) with reference to physical and mental health status, social relationships, and environmental and economic factors [2]. Physical disability in particular has been frequently associated with obesity due to its close link with the onset of debilitating co-morbidities (type 2 diabetes, hypertension, coronary artery disease, congestive heart failure, asthma, chronic back pain, osteoarthritis) or simply as a direct consequence in some individuals of an excess fat mass alongside a reduced lean body mass [3-7].

During the past decades there was an increasing prevalence of chronic disorders, due to improved social conditions, better preventative public health strategies, medical advancements, and overall extension of human lifespan. Chronic diseases, like obesity, are characterized by a slow progression and long duration, and, most importantly, rarely show a complete reversal to a normal physiological state. Therefore, the improvement of HRQoL in these conditions has become a primary therapeutic target [8] and patients' perspectives have gained more consideration by the medical community. HRQoL has become a primary target in evaluating the efficacy of the medical treatments and, in some cases, it has overcome healthrelated therapy targets [9]. The concept of quality of life (QoL) has been defined by the WHO as "individuals' perception of their position in life in the context of the culture and value systems in which they live and in relation to their goals, expectations, standards and concerns." In particular, the WHO values the perception of each individual of their QoL within the context of their daily living conditions $[10,11]$.

HRQoL can be classified in diverse domains (e.g., pain, physical, social, and functional impairment) and determined by different criteria (e.g., limitations in activities of daily living [ADL], difficulty in engaging in work or social interactions, limited mobility). It represents a key issue to be considered in the assessment and management of obesity since, on the one hand, all the HRQoL domains may be severely affected in these patients and, on the other, the perceived HRQoL may affect the clinical course and the outcome of treatments. 
Donini et al.: HRQoL Perceived by Subjects with Obesity

Our hypothesis is that clinical status and psychological symptoms can represent the main determinants of HRQoL associated with the presence of obesity. The aim of the present study is to evaluate the association between obesity and HRQoL and ascertain whether these associations are modified by the presence of comorbidities and abnormal psychological status.

\section{Methods}

\section{Recruitment and Sample Characteristics}

Between June 2012 and August 2016, 273 subjects with obesity (199 women and 74 men) were recruited among outpatients referred to the High Specialization Centre for the Care of Obesity (CASCO) at the Pathophysiology, Food Science and Endocrinology Section, Department of Experimental Medicine, Umberto I University Hospital (Sapienza University of Rome, Italy), and the San Camillo-Forlanini Hospital (Rome, Italy). They represent $45 \%$ of all potentially eligible subjects referred to the two clinical settings. Inclusion criteria were the ability to follow instructions to complete the questionnaires, body mass index (BMI) $\geq 30 \mathrm{~kg}$ / $\mathrm{m}^{2}$, and age $\geq 18$ and $<75$ years. There were no exclusion criteria concerning the presence of comorbidities (such as obstructive sleep apnoea, diabetes, or osteoarthritis) except for severe psychiatric disorders (e.g., schizophrenia, bipolar disorder) as assessed by psychologists included in the clinical team.

\section{Demographics and Clinical Status}

Medical history, with particular attention to obesity-related complications (insulin resistance, type 2 diabetes, hypertension, dyslipidaemia, thyroid dysfunction, and cardiovascular, osteoarticular, respiratory, gastrointestinal, and psychiatric diseases), was obtained. Comorbidity was defined as the sum of diseases affecting the patients.

\section{Anthropometric Measurements}

All measurements were performed following standardized procedures [12]. Body weight was measured using a SECA scale (Hamburg, Germany) to the nearest $0.1 \mathrm{~kg}$; height was measured using a SECA stadiometer (Hamburg, Germany) to the nearest $0.5 \mathrm{~cm}$. BMI (body weight/height squared) was calculated.

\section{Questionnaires}

1. The Italian version of the Laval questionnaire [13] was self-administered to evaluate the HRQoL. The Laval questionnaire consists of 44 items assessing 6 domains (symptoms, activity/mobility, personal hygiene/clothing, emotions, social interaction, and sexual life). Each domain is scored on a 7-point Likert scale, with higher scores corresponding to a better QoL [14]. Standardized Cronbach's $\alpha=0.87$ for symptoms, 0.9 for activity/ mobility, 0.88 for personal hygiene/clothing, 0.85 for emotions, 0.84 for social interactions, and 0.79 for sexual life [13].

2. TSD-OC (SIO test for obesity-related disabilities) is a reliable and validated instrument for measuring self-reported disability in subjects with obesity. The questionnaire consists of multiple questions measuring 7 dimensions (pain, stiffness, functionality and autonomy in daily activities, housework, outdoor activities, occupational activities, and social life). Each question is scored by the subject from 0 (lack of disability) to 10 (maximum disability). Standardized Cronbach's $\alpha=0.92$ [15].

3. SCL-90 (Symptom Checklist-90) is a self-reported psychometric questionnaire designed to evaluate several psychological problems and symptoms of psychopathology. It consists of 90 items, summarized in a Global Severity Index (GSI). The primary symptom dimen- 
Donini et al.: HRQoL Perceived by Subjects with Obesity

Table 1. Participants' clinical and demographic characteristics

\begin{tabular}{lcc}
\hline & Men & Women \\
\hline Study population, $n$ & 74 & 199 \\
Age, years & $46.2 \pm 14.2$ & $46.1 \pm 13.5$ \\
BMI, kg/m ${ }^{2}$ & $40.4 \pm 8.3$ & $34.8 \pm 6.2$ \\
\hline Obesity-related diseases, \% & & \\
Type 2 diabetes mellitus & 14.9 & 12.6 \\
Thyroid diseases & 6.8 & 35.2 \\
Gastrointestinal diseases & 23 & 19.6 \\
Cardiovascular diseases & 16.2 & 5.0 \\
Hypertension & 45.9 & 36.2 \\
Osteoarticular diseases & 25.7 & 22.1 \\
Respiratory diseases & 21.6 & 16.6 \\
Psychiatric diseases & 14.9 & 12.1 \\
Dyslipidaemia & 16.2 & 12.6 \\
Insulin resistance & 10.8 & 15.1 \\
\hline Education level, \% & & \\
Primary school & 2.7 & 5.0 \\
Middle school & 20.3 & 25.3 \\
High school & 60.8 & 55.1 \\
University degree & 16.2 & 14.6 \\
\hline Occupation, \% & & \\
Housewife & 1.4 & 32.5 \\
Employee & 21.9 & 29.6 \\
Freelance worker & 17.6 & \\
Factory worker & 5.9 & \\
Retired & & \\
Unemployed & & \\
Student & & \\
\hline & & \\
\hline & & \\
\hline
\end{tabular}

BMI, body mass index.

sions that are assessed are somatization, obsessive-compulsive, interpersonal sensitivity, depression, anxiety, hostility, phobic anxiety, and paranoid ideation. Standardized Cronbach's $\alpha=0.96[16]$.

\section{Statistics}

Data were entered in a Microsoft Excel database. Statistical analysis was performed using SPSS statistical software (IBM SPSS Statistics for Windows, released 2013; IBM Corp., Armonk, NY, USA).

First, a descriptive analysis of demographic and clinical characteristics was performed. Continuous variables were examined for skewness and kurtosis in order to test the normality assumptions. ANOVA were used for comparison of variables normally distributed while the Kruskal-Wallis test was used for variables not normally distributed (Shapiro-Wilk test $<0.05$ ).

A bivariate analysis (Spearman's rank correlation) was performed to test the association between the global scores of the Laval questionnaire (dependent variable) and age, BMI, comorbidity, and the global scores of TSD-OC and SCL-90. Furthermore, the correlation between the different dimensions of HRQoL defined through the Laval questionnaire (dependent variables) and age, BMI, together with the different dimensions of disability (TSD-OC) and psychological symptoms (SCL-90) was evaluated.

The relationship between the Laval global score and all the potential explanatory variables (each variable composing the TSD-OC and SCL-90 score) was analysed using, first of all, 
Donini et al.: HRQoL Perceived by Subjects with Obesity

Table 2. Bivariate analysis: correlation (Spearman's $\rho$ ) between Laval global score and other variables

\begin{tabular}{|c|c|c|c|c|}
\hline & \multicolumn{2}{|l|}{$\operatorname{Men}(n=74)$} & \multicolumn{2}{|c|}{ Women $(n=199)$} \\
\hline & Spearman's $\rho$ & $p$ & Spearman's $\rho$ & $p$ \\
\hline Age & -0.041 & 0.73 & -0.11 & 0.12 \\
\hline Number of diseases & -0.064 & 0.59 & -0.291 & $<0.001$ \\
\hline BMI & -0.442 & $<0.001$ & -0.4 & $<0.001$ \\
\hline \multicolumn{5}{|l|}{$S C L-90$} \\
\hline GSI & -0.591 & $<0.001$ & -0.7 & $<0.001$ \\
\hline Somatization & -0.539 & $<0.001$ & -0.742 & $<0.001$ \\
\hline Obsessive compulsive & -0.591 & $<0.001$ & -0.631 & $<0.001$ \\
\hline Interpersonal sensitivity & -0.467 & $<0.001$ & -0.57 & $<0.001$ \\
\hline Depression & -0.651 & $<0.001$ & -0.656 & $<0.001$ \\
\hline Anxiety & -0.489 & $<0.001$ & -0.607 & $<0.001$ \\
\hline Hostility & -0.418 & $<0.001$ & -0.456 & $<0.001$ \\
\hline Phobic anxiety & -0.448 & $<0.001$ & -0.53 & $<0.001$ \\
\hline Paranoid ideation & -0.419 & $<0.001$ & -0.496 & $<0.001$ \\
\hline \multicolumn{5}{|l|}{$T S D-O C$} \\
\hline Global score & -0.567 & $<0.001$ & -0.713 & $<0.001$ \\
\hline Pain & -0.472 & $<0.001$ & -0.626 & $<0.001$ \\
\hline Stiffness & -0.457 & $<0.001$ & -0.581 & $<0.001$ \\
\hline ADL & -0.54 & $<0.001$ & -0.638 & $<0.001$ \\
\hline Housework & -0.557 & $<0.001$ & -0.632 & $<0.001$ \\
\hline IADL & -0.447 & $<0.001$ & -0.621 & $<0.001$ \\
\hline Occupational activities & -0.382 & $<0.001$ & -0.542 & $<0.001$ \\
\hline Social life & -0.446 & $<0.001$ & -0.604 & $<0.001$ \\
\hline
\end{tabular}

SCL-90, Symptom Checklist-90; TSD-OC, Test SIO per le Disabilità Obesità Correlate (Italian Society of Obesity test for obesity-related disabilities); GSI, global severity index; ADL, activities of daily living; IADL, instrumental activities of daily living. No variable was distributed normally (Shapiro-Wilk test: $p<0.05$ ).

a block-wise linear regression model where all variables that were found to be individually significantly correlated with the outcome variable were entered in the model. Subsequently, a stepwise linear regression model with a forward selection ( $\alpha$-to-enter and $\alpha$-to-remove significance level $=0.05$ ) to define the best fitting model was performed. Collinearity diagnostics were carried out.

Data are presented as mean \pm SD or percentage, as appropriate. Differences were considered to be statistically significant when $p<0.05$.

\section{Results}

Demographic and clinical characteristics are summarized in Table 1. A total of 273 (74 men and 199 women) were enrolled. The mean age was $46.2 \pm 14.2$ and $46.1 \pm 13.5$ years, respectively, in men and women.

The correlation analysis is described in Tables 2 and 3. In the present sample standardized Cronbach's $\alpha$ were as follows: 0.85 for symptoms, 0.87 for activity/mobility, 0.9 for personal hygiene/clothing, 0.85 for emotions, 0.82 for social interactions, and 0.8 for sexual life. All the potential explanatory variables (except age, level of education, and occupation in both genders and the number of diseases in men) were significantly correlated $(p<0.05)$ with the Laval global score. In particular, HRQoL was negatively correlated with higher levels of 
Table 3. Laval score as a function of level of education and occupation

\begin{tabular}{|c|c|c|c|c|}
\hline & \multicolumn{2}{|l|}{$\operatorname{Men}(n=74)$} & \multicolumn{2}{|c|}{ Women $(n=199)$} \\
\hline & Laval score & $p$ & Laval score & $p$ \\
\hline \multicolumn{5}{|l|}{ Level of education } \\
\hline Primary school & $6.20 \pm 0.26$ & 0.39 & $4.63 \pm 1.37$ & 0.86 \\
\hline Middle school & $5.09 \pm 1.32$ & & $4.53 \pm 1.38$ & \\
\hline High school & $5.04 \pm 0.95$ & & $4.62 \pm 1.23$ & \\
\hline University degree & $5.3 \pm 0.69$ & & $4.42 \pm 1.47$ & \\
\hline \multicolumn{5}{|l|}{ Occupation } \\
\hline Employee & $4.87 \pm 1.21$ & 0.25 & $4.52 \pm 1.42$ & 0.22 \\
\hline Freelance worker & $5.20 \pm 0.70$ & & $4.75 \pm 1.18$ & \\
\hline Factory worker & $5.32 \pm 0.83$ & & $4.30 \pm 1.42$ & \\
\hline Retired & $5.31 \pm 0.78$ & & $3.94 \pm 1.13$ & \\
\hline Unemployed & $5.65 \pm 0.61$ & & $4.54 \pm 1.35$ & \\
\hline Student & $5.6 \pm 0.75$ & & $4.77 \pm 1.12$ & \\
\hline
\end{tabular}

Table 4. Linear regression model considering Laval global score

\begin{tabular}{|c|c|c|c|c|c|c|c|}
\hline $\begin{array}{l}\text { Dependent } \\
\text { variable }\end{array}$ & $\begin{array}{l}\text { Explanatory } \\
\text { variables }\end{array}$ & $\beta$ & SE & $\begin{array}{l}\text { Standard } \\
\text { coefficient } \beta\end{array}$ & $p$ & Model summary & \\
\hline \multirow{12}{*}{$\begin{array}{l}\text { Laval } \\
\text { global score }\end{array}$} & \multicolumn{7}{|c|}{ Block-wise regression analysis } \\
\hline & Age & -0.001 & 0.004 & -0.01 & 0.79 & $R^{2}$ & 0.59 \\
\hline & Comorbidity & 0.043 & 0.034 & 0.06 & 0.21 & Adjusted $R^{2}$ & 0.58 \\
\hline & BMI & $-0-03$ & 0.008 & -0.16 & $<0.001$ & SE of estimate & 0.8 \\
\hline & Education level & 0.004 & 0.066 & 0.002 & 0.95 & & \\
\hline & Occupation & 0.045 & 0.024 & 0.08 & 0.06 & & \\
\hline & SCL-90 GSI & -0.732 & 0.089 & -0.38 & $<0.001$ & & \\
\hline & TSD-OC global score & -2.375 & 0.272 & -0.45 & $<0.001$ & & \\
\hline & \multicolumn{7}{|c|}{ Stepwise regression analysis } \\
\hline & TSD-OC global score & -2.304 & 0.252 & -0.43 & $<0.001$ & $R^{2}$ & 0.58 \\
\hline & SCL-90 GSI & -0.733 & 0.088 & -0.38 & $<0.001$ & Adjusted $R^{2}$ & 0.58 \\
\hline & BMI & -0.029 & 0.007 & -0.16 & $<0.001$ & SE of estimate & 0.8 \\
\hline
\end{tabular}

SCL-90, Symptom Checklist-90; TSD-OC, Test SIO per le Disabilità Obesità Correlate (Italian Society of Obesity test for obesity-related disabilities); GSI, global severity index; BMI, body mass index.

BMI (Spearman's $\rho=-0.442$ and -0.4 , respectively, in men and women; $p<0.001$ ), higher levels of disability (Spearman's $\rho$ for TSD-OC global score $=-0.567$ and -0.713 , respectively, in men and women; $p<0.001$ ), and worse psychological problems (Spearman's $\rho$ for GSI at SCL-90 $=-0.591$ and -0.7 , respectively, in men and women; $p<0.001)$.

In Table 4, the regression model shows that BMI, disability (TSD-OC global score), and psychological symptoms (SCL-90 global severity index) were the main determinants of HRQoL defined by the global score of the Laval questionnaire. The proportion of the variance explained by the fully adjusted model was $58 \%$. The collinearity diagnostics showed no multicollinearity: all the eigenvalues were close to 1 (from 0.636 to 0.988 ), indicating that the predictors were poorly intercorrelated.

The correlation analysis exploring the association with the single domains of HRQoL defined by the Laval questionnaire (symptoms, activity/mobility, personal hygiene/clothing, emotions, social interaction, and sexual life) shows different patterns of associations but 
Donini et al.: HRQoL Perceived by Subjects with Obesity

some variables were consistently included in the regression models. In particular, BMI, TSD-OC pain, and TSD-OC social life were each included in more than 4 different domains of the Laval questionnaire (see online suppl. Tables 1-6; for all online suppl. material, see www. karger.com/doi/10.1159/000506079).

Age was significantly associated only with the "activity/mobility" and the "emotion" domains of the Laval questionnaire. SCL-90 depression domain contributed only to the "emotion" and the "social interaction" domains. Comorbidity influenced the "social interaction" and the "sexual life" domains while TSD-OC functionality and autonomy in daily activities was associated only with the "personal hygiene/clothing" and "activity/mobility" domains. SCL-90 interpersonal sensitivity was associated with 3 different HRQoL domains: "personal hygiene/clothing," "social interaction" and "sexual life." Occupation was associated with the "personal hygiene/clothing" and "emotion" dimensions and, finally, SCL-90 somatization was related only to the "symptoms" and the "activity/mobility" domains of the Laval questionnaire. Education and some SCL-90 symptoms (obsessive-compulsive, anxiety, hostility, phobic anxiety, paranoid ideation) and TSD-OC domains (stiffness, housework, outdoor activities, occupational activities) were not included in the stepwise regression model.

\section{Discussion}

The results of our study show that HRQoL was negatively correlated with higher levels of BMI, higher levels of disability, and worse psychological problems in patients with obesity seeking medical treatment. In addition, BMI, pain, and impairment in social life were found to be consistently related to most of the dimensions of HRQoL.

HRQoL refers to the self-reported effects of a medical condition like obesity on physical functioning and psychological and social well-being of patients. Obesity is associated with increased comorbidity and pain that can impair physical health status - functional limitations in daily activities that can increase the risk of disability, stigmatization, and discrimination which can affect mental well-being. Finally, it is generally accepted that obesity impairs HRQoL, and a greater severity of obesity is associated with greater impairment of QoL [17].

The assessment of HRQoL may be useful for researchers, clinicians, health managers, and policy makers. It offers a perspective into the current perception and feelings of an individual who is affected by a particular illness. The overall perceived impact of a particular condition on functioning and well-being may contribute to better define the phenotype of the patient beyond the information provided by medical and clinical measures. Moreover, the HRQoL measures can be considered as an outcome to evaluate the efficacy and cost-effectiveness of treatment interventions and the overall quality of the therapeutic approach and procedures. Finally, information on HRQoL may also contribute to the improvement of clinical pathways and procedures, health care spending, and public health policy [9].

In our study we used the validated Italian version of the Laval questionnaire [13]. HRQoL assessment may be performed through generic instruments (not specifically linked to a particular medical condition) that measure broad aspects of HRQoL (e.g., SF-36). This approach may allow comparisons of HRQoL across a variety of medical conditions in different populations. However, generic HRQoL instruments do not assess potential condition-specific domains of QoL that are more specifically affected by a specific disease. Another approach to HRQoL assessment is based on the use of instruments that are specifically designed and validated to a particular disease (e.g., osteoporosis), population (e.g., 
the elderly), or clinical problem (e.g., sexual function, pain). Disease-specific instruments are more powerful at detecting functional and psychological distress specifically correlated with a single disease and to assess treatment effects on QoL [9]. In fact, the use of disease-specific or generic instruments depends on the goals of the study. In this case we preferred a tool specifically designed for patients with obesity since we were interested, in particular, in the impact of the different elements that characterize the obesity phenotype on the different dimensions of QoL (symptoms, activity/mobility, personal hygiene/ clothing, emotions, social interaction, and sexual life) that can be analysed through the Laval questionnaire.

Obesity is a complex disease since it affects different dimensions: clinical comorbidity involving different organs and systems, functional impairment in ADL and in social interaction, and psychological wellbeing. The degree of involvement of each dimension may be different from one patient to another, thus creating a huge variety of bio-psycho-social phenotypes linked to obesity.

The concept of HRQoL is complex too. Different dimensions are involved. Physical, clinical, social, and psychological aspects may be encompassed in the concept of HRQoL, and thus the impairment of QoL may depend on different determinants and on the wide variability in individual responses to similar conditions [9].

Previous studies have generally focused on a global definition and grading of HRQoL without paying close attention to the relationship between the different phenotypes of obesity and the dimensions of QoL. In our study we tried to connect the different aspects of obesity (disability, psychological status, clinical and social aspects) to the diverse dimensions of HRQoL considered in the Laval questionnaire. This analysis allows a more precise definition of the determinants of reduced QoL in subjects with obesity, thus permitting a more focused intervention that can be more effective in improving QoL in obesity. It has been reported that physical problems in individuals with obesity affect HRQoL more than the mental problems. In fact, in our experience all the dimensions of QoL are affected by obesity with a reciprocal negative influence of each aspect on the others $[9,13,18]$.

In the present study all the dimensions of the Laval questionnaire were significantly affected (data not shown), thus confirming the overall impact of obesity on QoL. Few papers have analysed the determinants of HRQoL in subjects with obesity. In our study, disability (TSD-OC global score) and psychological symptoms (SCL-90 global severity index) were the main determinants of HRQoL defined by the global score obtained in the Laval questionnaire. In the study conducted by Warkentin et al. [19], increasing BMI, chronic pain (in particular due to fibromyalgia), depression, stroke, coronary disease, sleep apnoea, and less than fulltime employment were associated with reduced HRQoL measured using different instruments.

To the best of our knowledge, no study analysed the correlation of the single domains of HRQoL (symptoms, activity/mobility, personal hygiene/clothing, emotions, social interaction, and sexual life) and the single parameters describing the obesity phenotype. In our study, BMI, pain, and impairment in social life were found to be significant explanatory variables by most of the dimensions of HRQoL while age, comorbidity, psychological problems (depression, interpersonal sensitivity, somatization), and disability were involved only in some domains of the Laval questionnaire. Educational level together with different psychological symptoms (obsessive-compulsive, anxiety, hostility, phobic anxiety, paranoid ideation) and disability domains (stiffness, housework, outdoor activities, occupational activities) were not selected in the models for any dimension of the Laval questionnaire. These differences confirm the great variability in the manifestation of obesity in different subjects and above all the diverse impacts that single elements which characterize obesity can have on the determinism of QoL. The different aspects that define QoL are unequally influenced by the 
elements that characterize obesity (e.g., weight and fat mass, functional limitations, pain), and the various domains of QoL are perceived differently by subjects depending on psychological, clinical, and functional determinants.

The present study has some limitations. The items considered in the different tests (and in particular in the TSD-OC and the Laval questionnaires) may have a certain level of overlap by describing similar aspects. For example, the "personal hygiene/clothing" item of the Laval and the ADL domain of the TSD-OC may represent dimensions that can be attributed to the same functional limitation. On the other hand, each item emphasizes different aspects: personal hygiene and clothing, considered in the Laval questionnaire, do not represent all the ADLs contemplated by the TSD-OC. For this reason, we preferred to consider all the items in the three tests by taking into account a potentially overlapping level of evidence that did not seem to have affected the results. The study was conducted in a clinical sample of patients with obesity seeking help at a specialized clinical centre for their obesity problem. This means that the results could not be automatically translated to the general population living with obesity or to non-clinical samples.

In our study we did not disentangle the effects of obesity-related comorbidities (e.g., diabetes, osteoarthritis, obstructive sleep apnoea syndrome, anxiety, depression) on HRQoL. However, as happens in all chronic diseases, comorbidity is part of the overall definition of the disease, and all the clinical conditions that are involved in the definition of the overall picture of the disease have mutual interactions that are difficult (and perhaps unnecessary) to assess separately.

In conclusion, obesity is increasingly recognized as an important health problem. This study highlights the relationship of obesity with HRQoL. It also reports the association between different aspects of QoL and diverse components of the obesity phenotype. Targeting HRQoL may represent an important objective in the treatment of subjects with obesity. Since complete recovery from obesity is very difficult to achieve in the long term, it is important to value the results that can be obtained in terms of HRQoL to ensure that the patient is motivated to continue the treatment which is, however, long and tiring.

\section{Statement of Ethics}

Ethical approval and consent to participate: all procedures performed in studies involving human participants were in accordance with the ethical standards of the institutional and the national research committee and with the 1964 Helsinki Declaration and its later amendments or comparable ethical standards. The study protocol was approved by the Ethics Committee of Sapienza University of Rome. Oral and written informed consent was obtained from all participants included in the study.

\section{Disclosure Statement}

The authors declare that they have no competing interests.

\section{Funding Sources}

The study was supported by PRIN-RPNI (Research Project of National Interest) financed by the Ministry of Education, University and Research (MIUR) (protocol No. 2015JSWLTN). 
Donini et al.: HRQoL Perceived by Subjects with Obesity

\section{Author Contributions}

L.M.D. led the study design, was actively involved in the study conception, design, and strategic decisions, and drafted the manuscript. A.P. contributed to the study design, interpreted the findings, and helped in drafting the manuscript. A.R. performed the analysis of the data. L.D.L. assisted with data collection, entry, and analysis. C.L. and M.C. were closely involved in the study design, data collection, and field supervision. A.M.G., A.L., and M.S. participated in the study design and coordination and gave intellectual input on the manuscript. All authors read and approved the manuscript.

\section{Availability of Data and Material}

Please contact the authors for data requests.

\section{References}

1 World Health Organization. Obesity and overweight [cited 2018 August 17]. Available from: http://www.who. int/news-room/fact-sheets/detail/obesity-and-overweight.

2 Kolotkin RL, Andersen JR. A systematic review of reviews: exploring the relationship between obesity, weight loss and health-related quality of life. Clin Obes. 2017 0ct;7(5):273-89.

3 Beydoun MA, Beydoun HA, Wang Y. Obesity and central obesity as risk factors for incident dementia and its subtypes: a systematic review and meta-analysis. Obes Rev. 2008 May;9(3):204-18.

4 Jensen GL. Obesity and functional decline: epidemiology and geriatric consequences. Clin Geriatr Med. 2005 Nov;21(4):677-87.

5 Donini LM, Poggiogalle E, Mosca V, Pinto A, Brunani A, Capodaglio P. Disability affects the 6-minute walking distance in obese subjects (BMI $>40 \mathrm{~kg} / \mathrm{m}(2)$ ). PLoS One. 2013 Oct 11;8(10):e75491.

6 Donini LM, Merola G, Poggiogalle E, Lubrano C, Gnessi L, Mariani S, et al. Disability, physical inactivity, and impaired health-related quality of life are not different in metabolically healthy vs. unhealthy obese subjects. Nutrients. 2016 Nov;8(12):E759.

7 Poggiogalle E, Di Lazzaro L, Pinto A, Migliaccio S, Lenzi A, Donini LM. Health-related quality of life and quality of sexual life in obese subjects. Int J Endocrinol. 2014;2014:847871.

8 World Health Organization. Basic Documents. Geneva: WHO; 1948. Available from: https://apps.who.int/gb/ bd/PDF/bd48/basic-documents-48th-edition-en.pdf.

9 Fontaine KR, Barofsky I. Obesity and health-related quality of life. Obes Rev. 2001 Aug;2(3):173-82.

10 The World Health Organization Quality of Life assessment (WHOQOL): position paper from the World Health Organization. Soc Sci Med. 1995 Nov;41(10):1403-9.

11 Skevington SM, Lotfy M, O'Connell KA; WHOQOL Group. The World Health Organization's WHOQOL-BREF quality of life assessment: psychometric properties and results of the international field trial. A report from the WHOQOL group. Qual Life Res. 2004 Mar;13(2):299-310.

12 Lohman TG, Roche AF, Martorell R. Anthropometric standardization reference manual. Milan: EDRA; 1992. Available from: https://doi.org/10.1249/00005768-199208000-00020.

13 Donini LM, Rosano A, Di Lazzaro L, Poggiogalle E, Lubrano C, Migliaccio S, et al. Validation of the Italian version of the Laval questionnaire: health-related quality of life in subjects with obesity. Health Qual Life Outcomes. 2017 May;15(1):101.

14 Therrien F, Marceau P, Turgeon N, Biron S, Richard D, Lacasse Y. The laval questionnaire: a new instrument to measure quality of life in morbid obesity. Health Qual Life Outcomes. 2011 Aug;9(1):66.

15 Donini LM, Brunani A, Sirtori A, Savina C, Tempera S, Cuzzolaro M, et al.; SIO-ISDCA Task Force. Assessing disability in morbidly obese individuals: the Italian Society of Obesity test for obesity-related disabilities. Disabil Rehabil. 2011;33(25-26):2509-18.

16 Derogatis LR, Savitz KL. The SCL-90-R and the Brief Symptom Inventory (BSI) in primary care. In: Maruish ME, editor. Handbook of psychological assessment in primary care settings. Mahwah (NJ): Lawrence Erlbaum Associates; 2000. p. 297-334.

17 Hassan MK, Joshi AV, Madhavan SS, Amonkar MM. Obesity and health-related quality of life: a cross-sectional analysis of the US population. Int J Obes Relat Metab Disord. 2003 Oct;27(10):1227-32.

18 Jia H, Lubetkin EI. The impact of obesity on health-related quality-of-life in the general adult US population. J Public Health (Oxf). 2005 Jun;27(2):156-64.

19 Warkentin LM, Majumdar SR, Johnson JA, Agborsangaya CB, Rueda-Clausen CF, Sharma AM, et al. Predictors of health-related quality of life in 500 severely obese patients. Obesity (Silver Spring). 2014 May;22(5):1367-72. 